Published in final edited form as:

Biochem Soc Trans. 2012 February ; 40(1): 15-19. doi:10.1042/BST20110610.

\title{
Negative Cooperativity in the EGF Receptor
}

\author{
Linda J. Pike \\ Washington University School of Medicine Dept. of Biochemistry and Molecular Biophysics 660 \\ So. Euclid, Box 8231 St. Louis, MO 63110 Telephone: (314) 362-9502 FAX: (314) 362-7183 \\ pike@biochem.wustl.edu
}

\begin{abstract}
Scatchard analyses of the binding of EGF to its receptor yield concave up Scatchard plots, indicative of some type of heterogenity in ligand binding affinity. This was typically interpreted as being due to the presence of two independent binding site-one of high affinity representing $\leq 0 \%$ of the receptor population and one of low affinity making up the bulk of the receptors. However, the concept of two independent binding sites is difficult to reconcile with the X-ray structures of the dimerized EGF receptor that show symmetric binding of the two ligands. A new approach to the analysis of ${ }^{125}$ I-EGF binding data combined with the structure of the singly-occupied Drosophila EGF receptor have now shown that this heterogeneity is due to the presence of negative cooperativity in the EGF receptor. Concerns that negative cooperativity precludes ligandinduced dimerization of the EGF receptor confuse the concepts of linkage cooperativity. Linkage refers to the effect of ligand on the assembly of dimers while cooperativity refers to the effect of ligand binding to one subunit on ligand binding to the other subunit within a preassembled dimer. Binding of EGF to its receptor is positively linked with dimer assembly but shows negative cooperativity within the dimer.
\end{abstract}

\section{Keywords}

EGF receptor; saturation binding isotherms; negative cooperativity

\section{Early studies of the EGF receptor}

EGF was first purified from extracts of mouse submaxillary glands by Stanley Cohen nearly half a century ago, based on its ability to promote precocious eyelid opening and accelerate incisor eruption in newborn mice [1]. A decade later, receptors for EGF were identified by radioligand binding assay $[2,3]$. Although the earliest work showed linear Scatchard plots $[2,3]$, it very quickly became apparent that the Scatchard plots for the binding of EGF to its cell surface receptor exhibited upward concavity [4-6], indicating some type of heterogeneity in ligand binding affinity.

There are two possible interpretations for a concave up Scatchard plot. The first is that the system exhibits negative cooperativity, that is, binding of ligand to the first site in a dimer reduces the affinity of the second site for the ligand. The second explanation is that there are two independent binding sites of differing affinity. By definition, in a dimer showing negative cooperativity, half of the sites should be high affinity and half of the sites should be low affinity. However, in the case of the EGF receptor, only about $1 \%$ to $10 \%$ of the sites were high affinity sites $\left(\mathrm{K}_{\mathrm{D}} \sim 50 \mathrm{pM}\right)$. The vast majority of the sites were low affinity sites $\left(\mathrm{K}_{\mathrm{D}} \sim 3 \mathrm{nM}\right)$. Thus, the experimental data did not align with the predictions for negative cooperativity and the interpretation of curvilinear Scatchard plots as being due to the presence of "two independent classes of sites" became entrenched. 
Characterization of the EGF receptor protein proceeded apace and in 1980, Stanley Cohen's group at Vanderbilt reported that the EGF receptor co-purified with a protein kinase activity [7] that was subsequently shown to be a tyrosine kinase $[8,9]$. The receptor was cloned and sequenced in 1984 [10] and found to be comprised of a ligand binding extracellular domain, a single-pass transmembrane domain and an intracellular kinase domain. In 1987, Yarden and Schlessinger [11] showed that the EGF receptor existed in membranes as a monomer but dimerized upon addition of EGF. This resulted in the suggestion that the EGF receptor dimer represented the high affinity binding site while the monomer represented the low affinity site. However, since EGF induced its receptor to form dimers, there should have been positive cooperativity in this system-namely a concave down Scatchard plot-rather than negative cooperativity. Thus, it was clear that this simple model was inadequate for explaining the binding properties of the EGF receptor.

The first crystal structures of the doubly-liganded, dimerized extracellular domain appeared in 2002 [12, 13]. In 2003, the crystal structure of the effectively unliganded EGF receptor monomer was reported [14]. Together, they provide a clear picture of the effect of EGF binding on receptor dimerization. The extracellular domain of the EGF receptor is comprised of four subdomains, designated I through IV. In its monomeric form, the EGF receptor exists in a bent configuration, held together by intramolecular interactions between the so-called dimerization arm of subdomain II and the tethering arm of subdomain IV [14]. Upon binding EGF, this intramolecular tether is released and the receptor adopts an open configuration in which the dimerization arm is available to interact with the dimerization arm of a second receptor monomer. A back-to-back dimer is formed, mediated by receptorreceptor interactions $[12,13]$.

\section{Models of negative cooperativity}

Based on these structures, it was proposed that EGF bound with high affinity to the open or extended form of the receptor but with low affinity to the tethered form of the receptor. However, studies of the binding of EGF to receptor mutants [15] indicated that the extended form of the receptor did not represent the high affinity EGF binding site. Furthermore, mathematical modeling of the binding of EGF in a system in which a low affinity, tethered form of the receptor was in equilibrium with a high affinity extended form of the receptor could not recapitulate the in vivo binding data [16]. Instead, an unspecified "external" binding site that bound the receptor dimers with high affinity was invoked to fit the experimental data [16].

The reason that these modeling studies could not reproduce the binding properties of the EGF receptor was because the constraints applied to the model explicitly excluded negativity cooperativity [16]. Specifically, the affinity of EGF for binding to the second site on the dimer was constrained to be equal to the affinity of EGF for binding to the first site on the dimer. In fact, a decade earlier, Wofsy et al [17] had used the same model, but without these constraints, and showed that EGF binding data could theoretically be explained by the presence of negative cooperativity within the EGF receptor dimer. However, these workers did not provide experimental support for the larger predictions from this model.

\section{Negative cooperativity in a dimerizing system}

In the general model for the binding of EGF in dimerizing system (Figure 1), the unoccupied EGF receptor monomer is in equilibrium with the unoccupied EGF receptor dimer. This reaction is described by the association constant, $\mathrm{L}_{20}$. EGF can then bind to three different species: i) the monomer; ii) the first site on the dimer; and, iii) the second site on the dimer. The binding of EGF to the monomer is described by the association constant, $\mathrm{K}_{11}$. The 
binding of EGF to the first site on the dimer is described by the association constant, $\mathrm{K}_{21}$. And the binding of EGF to the second site on the dimer is described by the association constant, $\mathrm{K}_{22}$. As $\mathrm{L}_{21}$ and $\mathrm{L}_{22}$ are defined on the basis of microscopic equilibrium, only four parameters are needed to fully describe this model. Heterogeneity in ligand binding affinities derives from differences in $\mathrm{K}_{11}, \mathrm{~K}_{21}$ and $\mathrm{K}_{22}$.

For this model, the equation that describes the fractional saturation of the EGF receptor at any given concentration of EGF is:

$$
\bar{Y}=\frac{\mathrm{K}_{11}[\mathrm{EGF}]+\mathrm{L}_{20}[\mathrm{R}] \mathrm{K}_{21}[\mathrm{EGF}]\left[\left(1+2 \mathrm{~K}_{22}[\mathrm{EGF}]\right)\right]}{\left(1+\mathrm{K}_{11}[\mathrm{EGF}]\right)+2 \mathrm{~L}_{20}[\mathrm{R}]\left[1+\mathrm{K}_{21}[\mathrm{EGF}]\left(1+\mathrm{K}_{22}[\mathrm{EGF}]\right)\right]}
$$

where $[R]=$ concentration of unoccupied EGF receptor monomers [18]. In turn, the concentration of unoccupied EGF receptor monomers depends on the total concentration of EGF receptors in the cell, which can be calculated according to the equation derived by Wyman and Gill [19]:

$$
\mathrm{R}_{0}=[\mathrm{R}]\left(1+\mathrm{K}_{11}[\mathrm{EGF}]\right)+2 \mathrm{~L}_{20}[\mathrm{R}]^{2}\left(1+\mathrm{K}_{21}[\mathrm{EGF}]+\mathrm{K}_{21} \mathrm{~K}_{22}[\mathrm{EGF}]^{2}\right)
$$

If the affinity of EGF differs for the monomeric and dimeric forms of the receptor, this model predicts that the saturation binding isotherm for EGF will shift position depending on the total number of EGF receptors present in the cell. This is a simple consequence of mass action as more dimeric species will be present at high concentrations of receptor whereas more monomeric species will be present at low concentrations of receptor.

In 2008, we tested this prediction by putting the EGF receptor on a tetracycline-inducible promoter and stably transfecting it into $\mathrm{CHO}$ cells, which lack endogenous EGF receptors. We then performed ${ }^{125}$ I-EGF binding studies on cells induced to express increasing levels of EGF receptors [20]. Figure 2A shows an example of the data obtained in this type of experiment. The saturation binding isotherms shift from left to right with increasing numbers of EGF receptors per cell. Global modeling of the data from the all the curves yields a single set of fitted values for all four equilibrium constants, $\mathrm{K}_{11}, \mathrm{~K}_{21}, \mathrm{~K}_{22}$ and $\mathrm{L}_{20}$. The most striking finding from this analysis is that the affinity of EGF for the second site on the dimer $\left(\mathrm{K}_{22}\right)$ is substantially less than the affinity of EGF for the first site on the dimer $\left(\mathrm{K}_{21}\right)$. These data indicate that the general model for ligand binding in a dimerizing system is a valid description of EGF binding and demonstrate unequivocally that the binding of EGF to its receptor is negatively cooperative.

The existence of negative cooperativity in the EGF receptor would seem to be at odds with the demonstrated ability of the growth factor to induce the formation of receptor dimers. However, this logic is flawed because it fails to distinguish between the concepts of cooperativity and linkage. Cooperativity relates to the effect of the binding of ligand to one subunit in a preassembled dimer on the binding of ligand to the second subunit in that dimer. By contrast, linkage refers to the ability of a ligand to induce the assembly of dimers. Biochemical experiments indicate that the binding of EGF to its receptor induces dimer formation, ie. the EGF receptor exhibits positive linkage. This phenomenon is distinct from the negative cooperativity between subunits once the dimer is formed.

Positive linkage occurs when the affinity of a ligand for binding to the first site on the dimer is greater than that for binding to the monomer $\left(\mathrm{K}_{21}>\mathrm{K}_{11}\right)$. The values for $\mathrm{K}_{21}$ and $\mathrm{K}_{11}$ shown in the inset of Figure 2A are nearly identical suggesting that there is no linkage. 
However, this apparent absence of linkage is due to the fact that it is masked in the wild type receptor by the phosphorylation of the receptor that occurs subsequent to EGF binding. When the binding experiments are repeated in the kinase-dead K721A-EGF receptor (Figure 2B), the positive linkage become apparent [21]. Contrary to the situation in the wild type receptor, the saturation binding isotherms for the kinase-dead EGF receptor shift from right to left with increasing numbers of receptors per cell. Global fitting of the data reveals that negative cooperativity is still presents $\left(\mathrm{K}_{21}>\mathrm{K}_{22}\right)$ but in addition, positive linkage is present as $K_{21}$ is nearly two orders of magnitude greater than $K_{11}$. Thus, autophosphorylation of the receptor alters its ligand binding properties, masking the positive linkage that is present in the naive, unphosphorylated receptor.

The obvious differences between the binding characteristics of the wild type and kinasedead EGF receptors underscore the problem of relying on Scatchard plots for assessing the ligand binding properties of the EGF receptor. Both wild type and kinase-dead EGF receptors yield concave up Scatchard plots suggesting that the loss of kinase activity does not change the way EGF binds to its receptor. However, analysis of data from multiple saturation binding isotherms reveals that the binding properties of these two receptors are actually quite different. A second problem with the use of Scatchard plots arises when curvilinear plots are broken down into two 'independent' sites-ie. the high affinity and low affinity sites. In this situation, the values obtained for the number and affinity of the two classes of sites will vary depending on how many receptors are present in the cell. Thus, the data cannot be used to support claims for differences in the fraction of "high affinity sites" between two receptor mutants as the values obtained, even using the same receptor, will vary depending on receptor number. Finally, the underlying assumption in the derivation of the binding equations of which the Scatchard equation is a transformation, is that only a small fraction of the input ligand is bound. If the ligand binding assays are done under conditions in which more than $\sim 10 \%$ of the input ligand is bound, the Scatchard equation cannot be used to analyze the binding data [22]. Scatchard plots of such data show downward concavity, which is an artifact of the "over-binding" of ligand.

\section{Structural Basis for Negative Cooperativity}

By performing binding analyses on cells expressing increasing levels of various mutant forms of the EGF receptor, it is possible to obtain information on the structural requirements of the receptor for supporting negative cooperativity. Subdomain IV of the extracellular domain contains the tethering arm that is thought to interact with the dimerization arm in subdomain II to hold the EGF receptor monomer in its closed conformation [14]. Mutation of the three residues thought to be involved in this tethering interaction, Asp563, His566 and Lys585, does not significantly alter the ligand binding properties of the receptor [23]. This suggests that the tethering interactions are not involved in the allosteric regulation of ligand binding.

The tethering arm in subdomain IV contains two disulfide loops: i) Cys558-Cys567; and, ii) Cys571-Cys593. Deletion of the entire Cys558-Cys567 loop had only a modest effect on cooperativity and linkage in the EGF receptor. Similarly, release of this disulfide by substitution of the cysteine residues with alanines also had little effect on allostery in ligand binding. By contrast, deletion of the entire Cys571-Cys593 loop abrogated linkage and cooperativity in ligand binding. Release of this loop by double alanine substitution resulted in enhanced negative cooperativity [23]. These findings link the extracellular juxtamembrane domain, and particularly the region between Cys571 and Cys593 to negative cooperativity. 
Nested truncations from the C-terminus of the EGF receptor have also implicated the intracellular juxtamembrane domain in negative cooperativity [21]. Truncation of the Cterminal tail of the receptor (c'973-EGFR) yielded values for the four equilibrium association constants that were similar to those for the kinase-dead receptor and indicated the presence of both positive linkage and negative cooperativity. Surprisingly, deletion of the kinase domain as well as the C-terminal tail of the EGF receptor (c'698-EGFR) also yielded a receptor with intact positive linkage and negative cooperativity. By contrast, deletion of the entire intracellular domain led to the production of a receptor that showed neither cooperativity nor linkage. These findings suggest that the intracellular juxtamembrane domain of the EGF receptor is structurally involved in the genesis of negative cooperativity. Consistent with this interpretation is the finding that substitution of residues 647 and 650, just beyond the membrane, with cysteines, which enables palmitoylation of the receptor, also led to the complete loss of negative cooperativity [21]. Thus, it appears that the membrane-proximal portion of both the extracellular and intracellular domains of the EGF receptor are involved in generating negative cooperativity.

Recently, Alvarado et al. reported an X-ray crystal structure of the Drosophila EGF receptor, which yielded important insights into the structural basis for negative cooperativity in that receptor system [24]. In contrast to the human EGF receptor, which does not retain negative cooperativity when purified and assayed as the soluble extracellular domain, the isolated extracellular domain of the Drosophila EGF receptor (dEGFR) does show negative cooperativity [24]. The crystal structure of the singly-liganded dimer of the dEGFR shows an asymmetric dimer in which the second, unoccupied site is conformationally restrained and thus would have a significantly lower affinity for ligand than does the first site. Given the homologies between the human and Drosophila EGF receptors, it seems likely that the human EGF receptor also forms an asymmetric, singly-ligated extracellular domain dimer with an important difference being that the intracellular juxtamembrane domain of the human EGF receptor also contributes to this asymmetry.

\section{Conclusions}

Novel analyses of ligand binding data have demonstrated that the observed heterogeneity of EGF binding affinity results from negative cooperativity [20, 21, 23]. High affinity binding occurs to the first site on the receptor dimer whereas low affinity binding occurs to the second site on the dimer as well as to the monomer. The existence of negative cooperativity in the EGF receptor is further supported by the X-ray crystallographic structure of the Drosophila EGF receptor [24]. Additional studies are needed to explicitly define the structural basis of negative cooperativity in the mammalian EGF receptor and to determine how it contributes to the regulation of cell signaling.

\section{Acknowledgments}

This work was supported by National Institute of Health grants R01GM64491 and R01GM82824 to LJP.

\section{Abbreviations}

EGF epidermal growth factor

\section{References}

1. Cohen S. Isolation of a Mouse Submaxillary Gland Protein Accelerating Incisor Eruption and Eyelid Opening in the New-born Animal. J. Biol. Chem. 1962; 237:1555-1562. [PubMed: 13880319] 
2. Carpenter G, Lembach KJ, Morrison MM, Cohen S. Characterization of the Binding of 125ILabeled Epidermal Growth Factor to Human Fibroblasts. J. Biol. Chem. 1975; 250:4297-4304. [PubMed: 1126952]

3. Hollenberg MD, Cuatrecasas P. Epidermal Growth Factor: Recetors in Human Fibroblasts and Modulation of Action by Cholera Toxin. Proc. Natl. Acad. Sci. U.S.A. 1973; 70:2964-2968. [PubMed: 4355377]

4. King AC, Cuatrecasas P. Resolution of High and Low Affinity Epidermal Growth Factor Receptors. J. Biol. Chem. 1982; 257:3053-3060. [PubMed: 6277923]

5. Magun BE, Matrisian LM, Bowden GT. Epidermal Growth Factor. Ability of Tumor Promoter to Alter its Degradation, Receptor Affinity and Receptor Number. J. Biol. Chem. 1980; 255:63736381. [PubMed: 6967066]

6. Shoyab M, DeLarco JE, Todaro GJ. Biologically Active Phorbol Esters Specifically Alter Affinity of Epidermal Growth Factor Membrane Receptors. Nature. 1979; 279:387-391. [PubMed: 16068160]

7. Cohen S, Carpenter G, King L Jr. Epidermal Growth Factor-Receptor-Protein Kinase Interactions. Co-Purification of Receptor and Epidermal Growth Factor-Enhanced Phosphorylation Activity. J. Biol. Chem. 1980; 255:4834-4842. [PubMed: 6246084]

8. Ushiro H, Cohen S. Identification of Phosphotyrosine as a Product of Epidermal Growth FactorActivated Protein Kinase in A-431 Cell Membranes. J. Biol. Chem. 1980; 255:8363-8365. [PubMed: 6157683]

9. Hunter T, Cooper JA. Epidermal Growth Factor Induces Rapid Tyrosine Phosphorylation of Proteins in A431 Human Tumor Cells. Cell. 1981; 24:741-752. [PubMed: 6166387]

10. Ullrich A, Coussens L, Hayflick JS, Dull TJ, Gray A, Tam AW, Lee J, Yarden Y, Libermann TA, Schlessinger J, Downward J, Mayes ELV, Whittle N, Waterfield MD, Seeburg PH. Human Epidermal Growth Factor Receptor cDNA Sequence and Aberrant Expression of the Amplified Gene in A431 Epidermoid Carcinoma Cells. Nature. 1984; 309:418-425. [PubMed: 6328312]

11. Yarden Y, Schlessinger J. Epidermal Growth Factor Induces Rapid, Reversible Aggregation of the Purified Epidermal Growth Factor Receptor. Biochemistry. 1987; 26:1443-1451. [PubMed: 3494473]

12. Garrett TPJ, McKern NM, Lou M, Elleman TC, Adams TE, Lovrecz GO, Zhu H-J, Walker F, Frenkel MJ, Hoyne PA, Jorissen RN, Nice EC, Burgess AW, Ward CW. Crystal Structure of a Truncated Epidermal Growth Factor Receptor Extracellular Domain Bound to Transforming Growth Factor a. Cell. 2002; 110:763-773. [PubMed: 12297049]

13. Ogiso H, Ishitani R, Nureki O, Fukai S, Yamanaka M, Kim J-H, Saito K, Sakamoto A, Inoue M, Shirouzu M, Yokoyama S. Crystal Structure of the Complex of Human Epidermal Growth Factor and Receptor Extracellular Domains. Cell. 2002; 110:775-787. [PubMed: 12297050]

14. Ferguson KM, Berger MB, Mendrola JM, Cho H-S, Leahy DJ, Lemmon MA. EGF Activates Its Receptor by Removing Interactions that Autoinhibit Ectodomain Dimerization. Mol. Cell. 2003; 11:507-517. [PubMed: 12620237]

15. Mattoon D, Klein P, Lemmon MA, Lax I, Schlessinger J. The Tethered Configuration of the EGF Receptor Extracellular Domain Exerts Only a Limited Control of Receptor Function. Proc. Natl. Acad. Sci. U.S.A. 2004; 101:923-928. [PubMed: 14732693]

16. Klein P, Mattoon D, Lemmon MA, Schlessinger J. A Structure-Based Model for Ligand Binding and Dimerization of EGF Receptors. Proc. Natl. Acad. Sci. U.S.A. 2004; 101:929-934. [PubMed: 14732694]

17. Wofsy C, Goldstein B, Lund K, Wiley HS. Implications of Epidermal Growth Factor (EGF) Induced EGF Receptor Aggregation. Biophys. J. 1992; 63:98-110. [PubMed: 1420877]

18. Wong I, Lohman TM. Linkage of Protein Assembly to Protein-DNA Binding. Meth. Enzymol. 1995; 259:95-127. [PubMed: 8538477]

19. Wyman, J.; Gill, SJ. Binding and Linkage. Functional Chemistry of Biological Macromolecules. University Science Books; Mill Valley, CA: 1990.

20. Macdonald JL, Pike LJ. Heterogeneity in EGF Binding Affinities Arises From Negative Cooperativity in an Aggregating System. Proc. Natl. Acad. Sci. U.S.A. 2008; 105:112-117. [PubMed: 18165319] 
21. Macdonald-Obermann JL, Pike LJ. The Intracellular Juxtamembrane Domain of the EGF Receptor is Responsible for the Allosteric Regulation of EGF Binding. J. Biol. Chem. 2009; 284:13, 570$513,576$.

22. Swillens S. Interpretation of Binding Curves Obtained with High Receptor Concentrations: Practical Aid for Computer Analysis. Mol. Pharm. 1995; 47:1197-1203.

23. Adak S, Pike LJ. The Tethering Arm of the EGF Receptor is Required for Negative Cooperativity and Signal Transduction. J. Biol. Chem. 2011; 286:1545-1555. [PubMed: 21047778]

24. Alvarado D, Klein DE, Lemmon MA. Structural Basis for Negative Cooperativity in Growth Factor Binding to an EGF Receptor. Cell. 2010; 142:568-579. [PubMed: 20723758] 


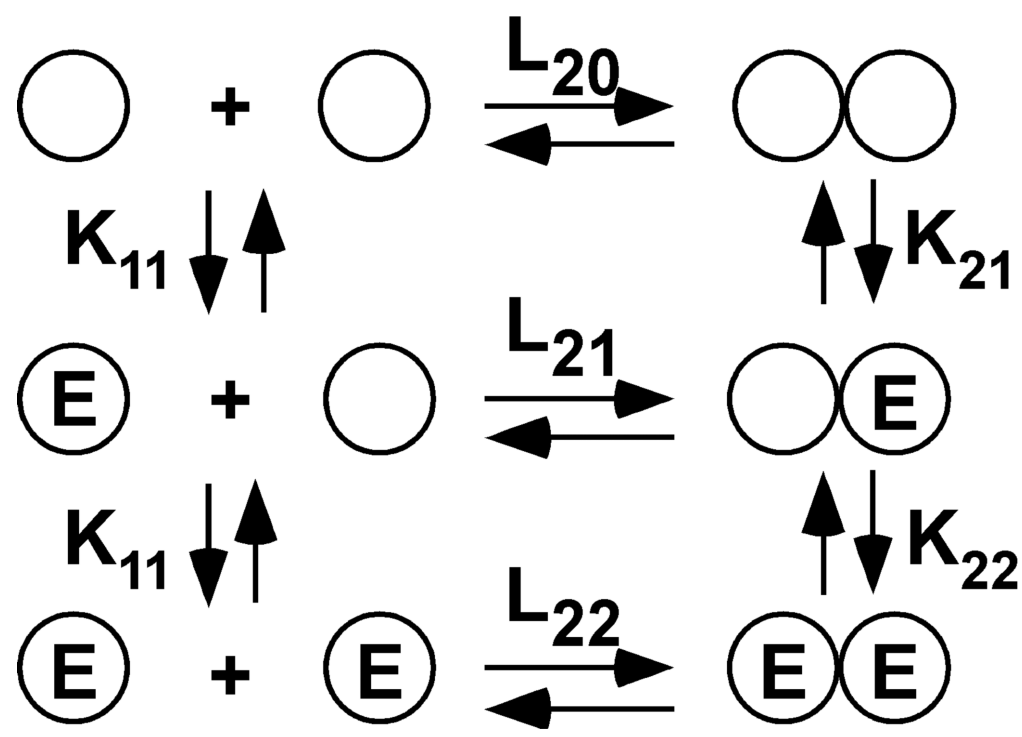

Figure 1. General model for the binding of ligand in a dimerizing system Open circles represent unoccupied EGF receptor monomers. E represents EGF. A circle containing an E represents a ligand-occupied EGF receptor. See text for further discussion. 


\section{A. Wild type-EGFR}

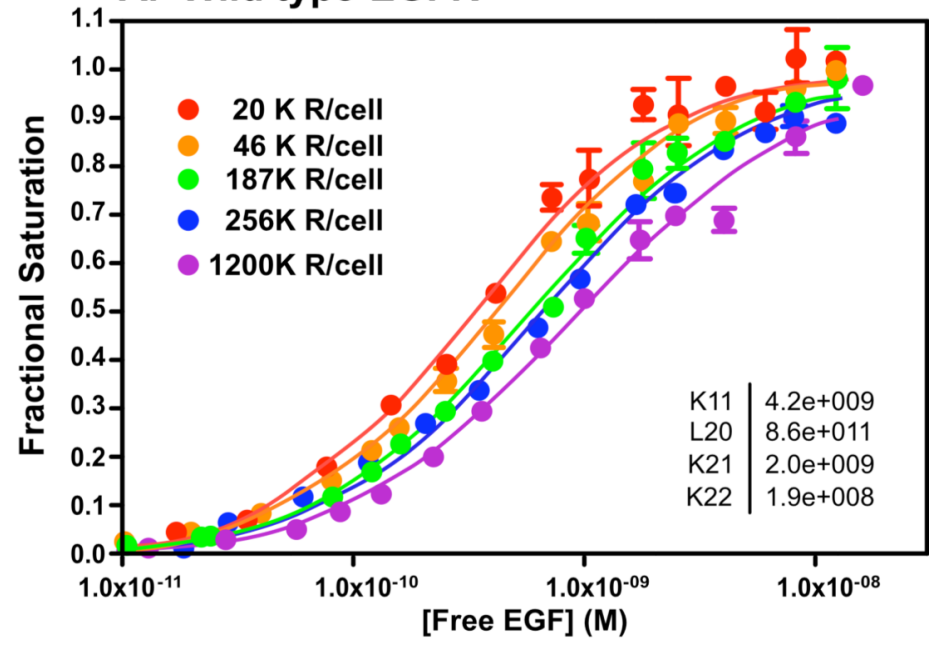

B. K721A-EGFR

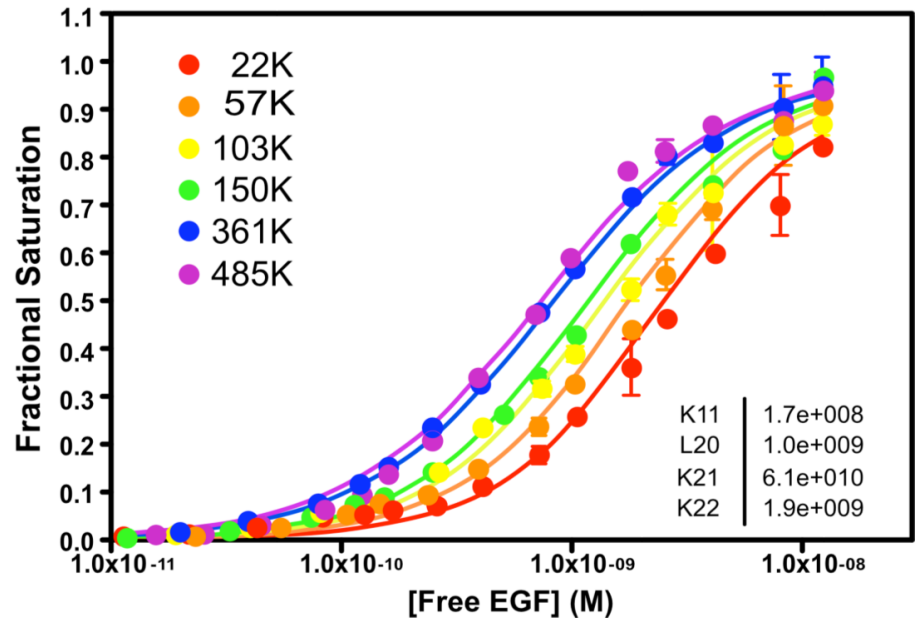

Figure 2. ${ }^{125}$ I-EGF binding to CHO cells expressing wild type or kinase-dead EGF receptors A) $\mathrm{CHO}$ cells were stably transfected with a plasmid expressing wild type EGF receptor from a tet-inducible promoter. Cells were cultured for $48 \mathrm{hr}$ with increasing concentrations of doxycycline to induce receptor expression. ${ }^{125}$ I-EGF binding was then carried out by incubating cells overnight at $4^{\circ} \mathrm{C}$ with $40 \mathrm{pM}^{125} \mathrm{I}-\mathrm{EGF}$ and increasing concentrations of unlabeled EGF. B) Same as A) except that $\mathrm{CHO}$ cells were expressing the kinase.dead K721A-EGF receptor from the tet-inducible promoter. This research was originally published in the Journal of Biological Chemistry. (Macdonald-Obermann, J. L. and Pike, L. J., The Intracellular Juxtamembrane Domain of the Epidermal Growth Factor Receptor is Responsible for the Allosteric Regulation of EGF Binding. 